\title{
Serum levels of hyaluronic acid are associated with COPD severity and predict survival
}

\author{
Eleni Papakonstantinou ${ }^{1,2}$, Ioannis Bonovolias ${ }^{2}$, Michael Roth ${ }^{1}$, Michael Tamm¹, \\ Desiree Schumann ${ }^{1}$, Florent Baty ${ }^{3}$, Renaud Louis ${ }^{4}$, Branislava Milenkovic ${ }^{5}$, \\ Wim Boersma ${ }^{6}$, Bram Stieltjes $^{7}$, Konstantinos Kostikas ${ }^{1}$, Francesco Blasi ${ }^{8}$, \\ Joachim G. Aerts ${ }^{9}$, Gernot G.U. Rohde (10 ${ }^{10}$, Alicia Lacoma (1) ${ }^{11}$, Antoni Torres ${ }^{12}$, \\ Tobias Welte ${ }^{13}$ and Daiana Stolz ${ }^{1}$
}

@ERSpublications

Serum hyaluronic acid (HA) is associated with COPD severity, outcome and predicts overall survival. Enzymatic degradation of $\mathrm{HA}$ is associated with airflow limitation and impairment of lung function. http://ow.ly/COl830n2IcQ

Cite this article as: Papakonstantinou E, Bonovolias I, Roth M, et al. Serum levels of hyaluronic acid are associated with COPD severity and predict survival. Eur Respir J 2019; 53: 1801183 [https://doi.org/ 10.1183/13993003.01183-2018].

ABSTRACT Hyaluronic acid (HA) and its degradation products play an important role in lung pathophysiology and airway remodelling in chronic obstructive pulmonary disease (COPD).

We investigated if HA and its degrading enzyme hyaluronidase (HYAL)-1 are associated with COPD severity and outcome.

Serum HA was assessed in a discovery cohort of 80 COPD patients at stable state and exacerbations. HA, HYAL-1 and HYAL-1 enzymatic activity were evaluated at stable state, exacerbations and 4 weeks after exacerbations in 638 COPD patients from the PROMISE validation cohort.

In the discovery cohort, serum HA was higher at exacerbations compared with the stable state $(\mathrm{p}=0.015)$. In the validation cohort, HA was higher at moderate and severe exacerbations than at baseline $(\mathrm{p}<0.001)$, and remained higher after 4 weeks $(\mathrm{p}<0.001)$. HA was strongly predictive for overall survival since it was associated with time to death $(\mathrm{p}<0.001)$ independently of adjusted Charlson score, annual exacerbation rate and BODE (body mass, airflow obstruction, dyspnoea, exercise capacity) index. Serum HYAL-1 was increased at moderate $(\mathrm{p}=0.004)$ and severe $(\mathrm{p}=0.003)$ exacerbations, but decreased after 4 weeks $(p<0.001)$. HYAL-1 enzymatic activity at stable state was inversely correlated with FEV1\% pred $(\mathrm{p}=0.034)$ and survival time $(\mathrm{p}=0.017)$.

Serum HA is associated with COPD severity and predicts overall survival. Degradation of HA is associated with airflow limitation and impairment of lung function. 


\section{Introduction}

Chronic obstructive pulmonary disease (COPD) is a complex heterogeneous disease characterised by the progressive development of airflow limitation that is largely irreversible. Acute exacerbations of COPD may occur at any stage of COPD, and are associated with patients' mortality and morbidity $[1,2]$.

A key feature of COPD is airway remodelling, which describes the serious alterations of the extracellular matrix (ECM) affecting airway wall thickness, resistance and elasticity [3]. The ECM consists of a dynamic combination of structural molecules such as collagens and glycosaminoglycans. Hyaluronic acid (HA) is a ubiquitous, naturally occurring glycosaminoglycan that is found in high concentrations in mammalian connective tissue, including the lung $[4,5]$. HA is synthesised by the action of HA synthases and it is degraded by hyaluronidases (HYALs) $[6,7]$. There is emerging evidence that HA and its degradation products play an important role in lung pathophysiology.

The biological functions of HA depend on its molecular size. High-molecular-weight HA (>1000 kDa), which is the physiologically available form, promotes cell survival, and has anti-inflammatory, anti-angiogenic and immunosuppressive effects. In contrast, low-molecular-weight HA $(150-350 \mathrm{kDa})$ is produced during inflammation; it promotes cell migration, and has pro-inflammatory and pro-angiogenic properties [8-12].

Studies in animal models of inflammatory lung conditions and in patients with asthma suggest a potential role for high-molecular-weight HA as a treatment for airway diseases [13-15]. In mouse models of COPD, aerosolised HA protects endogenous HA in lung tissue against degradation by cigarette smoke-generated reactive oxygen species, and reduces air space enlargement and mitigation of elastic fibre injury [13].

We have previously shown that in airway smooth muscle cells from patients with COPD, HA metabolism is impaired and characterised by decreased synthesis of HA, associated with downregulation of HA synthase-1 and upregulation of HYAL-1 [16]. Furthermore, acute exacerbations of COPD are associated with increased HYAL activity in bronchoalveolar lavage and subsequent degradation of HA, which may contribute to airway inflammation and subsequent lung function decline during exacerbations [17]. In addition to HA, degradation products of other ECM molecules such as collagen and elastin are also associated with clinically relevant outcomes in COPD [18].

In the present study, we analysed serum levels of HA and HYAL-1 in two well-matched cohorts of COPD patients. We hypothesise that HA is associated with COPD progression and severity, and predicts overall survival in COPD.

\section{Methods}

\section{Study design and participants}

A two-stage study design was used to minimise unnecessary waste of patient samples. Initial analysis was performed in a discovery cohort of 80 COPD patients with either stable disease $(n=65)$ or at exacerbation $(\mathrm{n}=15)$ included in the BASCO study (Basel Study on COPD), a cross-sectional, observational, monocentric study (figure 1). The discovery analysis was limited to assessment of serum levels of HA and was undertaken before the complete detailed analysis of a validation cohort consisting of 638 patients that were included in the PROMISE-COPD cohort (Predicting Outcome using Systemic Markers in Severe Exacerbations of Chronic Obstructive Pulmonary Disease; ISRCTN Registry identifier ISRCTN99586989) (figure 1). The PROMISE-COPD study was an investigator-driven, multicentre, longitudinal trial performed at 11 tertiary respiratory centres in eight European countries. BASCO and PROMISE-COPD complied with the Declaration of Helsinki and Good Clinical Practice guidelines, and were approved by

Affiliations: ${ }^{1}$ Clinic of Pulmonary Medicine and Respiratory Cell Research, University Hospital, Basel, Switzerland. ${ }^{2}$ Dept of Pharmacology, School of Medicine, Aristotle University of Thessaloniki, Thessaloniki, Greece. ${ }^{3}$ Pneumology, Kantonsspital St Gallen, St Gallen, Switzerland. ${ }^{4}$ Dept of Pneumology, CHU Liege, University of Liege, GIGAI Research Group, Liege, Belgium. ${ }^{5}$ Faculty of Medicine, University of Belgrade Clinic for Pulmonary Diseases, Belgrade, Serbia. ${ }^{6}$ Dept of Pneumology, Medisch Centrum Alkmaar, Alkmaar, The Netherlands. ${ }^{7}$ Clinic of Radiology and Nuclear Medicine, University Hospital Basel, Basel, Switzerland. ${ }^{8}$ Internal Medicine Dept, Respiratory Unit and Adult Cystic Fibrosis Center Fondazione IRCCS Cà Granda Ospedale Maggiore Policlinico Milano, Dept of Pathophysiology and Transplantation, Università degli Studi di Milano, Milan, Italy. ${ }^{9}$ Dept of Pneumology, Amphia Hospital/Erasmus MC, Breda, The Netherlands. ${ }^{10}$ Dept of Respiratory Medicine, Maastricht University Medical Center, Maastricht, The Netherlands. ${ }^{11}$ Dept of Microbiology, Hospital Universitari Germans Trias i Pujol, CIBER Enfermedades Respiratorias, Badalona, Spain. ${ }^{12}$ Dept of Pneumology, Hospital Clinic, Barcelona, Spain. ${ }^{13}$ Dept of Pneumology, Medizinische Hochschule Hannover, Hannover, Germany.

Correspondence: Daiana Stolz, Clinic of Pulmonary Medicine and Respiratory Cell Research, University Hospital of Basel, Petersgraben 4, 4031 Basel, Switzerland. E-mail: daiana.stolzQuusb.ch 


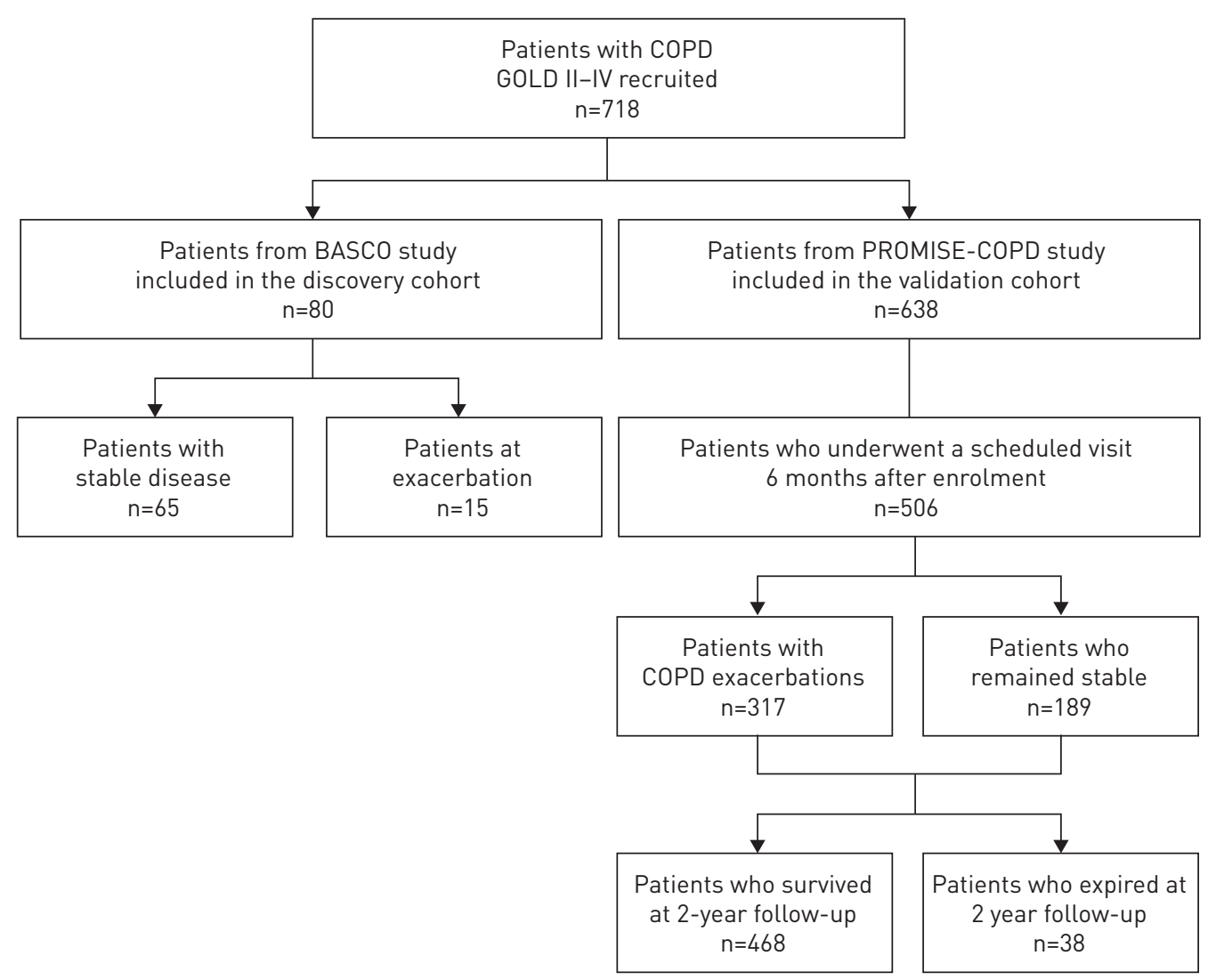

FIGURE 1 Study design. COPD: chronic obstructive pulmonary disease; GOLD: Global Initiative for Chronic Obstructive Lung Disease. A discovery cohort of 80 patients was drawn from the cohort of the BASCO study. The validation cohort was comprised of 638 patients that were included in the PROMISE-COPD study. GOLD classification II-IV corresponds to moderate to very severe COPD.

the Institutional Review Board of the University of Basel (EKBB05/06 and EKBB295/07). All the patients provided prior written consent for the study assessments.

For the validation cohort, we consecutively recruited 638 patients that, at baseline, appeared with clinically stable, moderate to very severe COPD (forced expiratory volume in $1 \mathrm{~s} \mathrm{(FEV1)} \mathrm{\%} \mathrm{pred}<80 \%$ and $\mathrm{FEV}$ //forced vital capacity $(\mathrm{FVC}) \leqslant 0.7$ ), based on physical examination and spirometry, at least 4 weeks after the latest exacerbation was resolved [19-21]. All patients were $\geqslant 40$ years old with a smoking history $\geqslant 10$ pack-years. Exclusion criteria were rapid fatal disease with death expected within 6 months, pulmonary condition other than COPD as the main respiratory disease (e.g. bronchiectasis, asthma or pulmonary fibrosis), immunosuppression including organ transplantation or chronic steroid use ( $>20 \mathrm{mg} \cdot \mathrm{day}^{-1}$ prednisolone equivalent), or musculoskeletal or neuromuscular process preventing ambulation.

At baseline, patients underwent clinical evaluation, sputum analysis and standard spirometry after the administration of an inhaled short-acting bronchodilator. The severity of obstruction and condition of the patients was graded according to the stages of disease defined by the Global Initiative for Chronic Obstructive Lung Disease (GOLD) and divided into GOLD groups II-IV. Each patient underwent the 6-min walk test, and provided information on quality of life using the St George's Respiratory Questionnaire (SGRQ) and the Short Form-36 questionnaire. Furthermore, the modified Medical Research Council dyspnoea score, BODE (body mass, airflow obstruction, dyspnoea, exercise capacity) index and age-adjusted Charlson score were determined for each patient. Comorbidities including associated medical therapy were assessed at baseline, at exacerbation and at biannual visits. Non-contrast-enhanced multislice computed tomography (CT) imaging data sets were acquired in 150 patients at inspiratory breath-hold. From these data sets, the lung volume was extracted automatically using open-source software that was developed in-house and based on ImageJ [22]. From these data, lung volume and previously described histogram-based density value distributions including the low-attenuation area <-950 HU (\%) and adjusted lung density ( $\mathrm{g} \cdot \mathrm{L}^{-1}$ ) were extracted and assessed to automatically quantify emphysema [23]. After the baseline visit, patients had scheduled visits every 6 months, and unscheduled visits at exacerbation and 4 weeks later. The median follow-up was 24 months. 
For patients included in the discovery cohort, serum samples, at stable state or at exacerbation, were collected at the day of bronchoscopy. In the validation cohort, serum samples were collected at stable state, at exacerbation (moderate and severe) and at follow-up (4 weeks after exacerbation).

The diagnosis of stable COPD and of exacerbations was made according to GOLD guidelines. According to the standard definition, an exacerbation of COPD was diagnosed as an acute event characterised by a worsening of the patient's respiratory symptoms beyond normal day-to-day variations and which leads to a change in medication. Generally, patients reported a deterioration of one or more of the following typical symptoms: increased frequency and severity of cough, increased sputum production, change in sputum colour, and/or increased dyspnoea. COPD exacerbations were defined as moderate (requiring either antibiotics and/or parenteral corticosteroids) or severe (requiring hospitalisation or resulting in death) [2].

\section{Measurement of HA and HYAL-1 in the serum of COPD patients}

HA was measured in aliquots of serum obtained from COPD patients of the discovery cohort at stable state and at exacerbation, and from COPD patients of the validation cohort at stable state, at exacerbation and 4 weeks after an exacerbation using an HA binding protein-based sandwich ELISA (Corgenix, Westminster, CO, USA). Intra-assay coefficient of variation: $\leqslant 7.7 \%$; inter-assay coefficient of variation: $\leqslant 8.3 \%$.

HYAL-1 was measured in aliquots of serum obtained from COPD patients of the validation cohort at stable state, at exacerbation and 4 weeks after an exacerbation using a sandwich ELISA (Wuhan EIAab Science, Wuhan, China). Intra-assay coefficient of variation: $\leqslant 4.3 \%$; inter-assay coefficient of variation: $\leqslant 7.5 \%$.

\section{HYAL activity in serum}

HYAL activity was measured in aliquots of serum ( $5 \mu \mathrm{L}$ from diluted serum 1/100) obtained from COPD patients of the validation cohort at stable state by HA substrate zymography on $10 \%$ polyacrylamide gels containing $0.17 \mathrm{mg} \cdot \mathrm{mL}^{-1} \mathrm{HA}$ (Sigma, St Louis, MO, USA). Gels were washed with $0.1 \mathrm{M} \mathrm{HCOONa}$ buffer, $0.03 \mathrm{M} \mathrm{NaCl}$ and 2.5\% Triton $\mathrm{X}-100, \mathrm{pH} 4.0$, for $1 \mathrm{~h}$ at room temperature, and then incubated in $0.1 \mathrm{M}$ formic acid buffer and $0.03 \mathrm{M} \mathrm{NaCl}, \mathrm{pH} 4.0$, for $20 \mathrm{~h}$ at $37^{\circ} \mathrm{C}$. After washing with $20 \%$ ethanol/10\% acetic acid for $20 \mathrm{~min}$, gels were stained with $0.5 \%$ Alcian blue in $20 \%$ ethanol $/ 10 \%$ acetic acid, and destained with the same solution without the dye. The HA lysis bands were quantified using a computer-assisted image analysis program (Eastman Kodak, Rochester, NY, USA).

\section{Determination of markers of ECM turnover}

Serum levels of markers of ECM turnover such as degradation fragments of collagen types I, IV ( $\alpha_{1}$ chain and $\alpha_{3}$ chain) and VI, pro-forms of collagen types III, V and VI, and neutrophil elastase were measured with Nordic Bioscience (Herlev, Denmark) assays, as previously described [18, 24].

\section{Statistical analysis}

SPSS for Windows version 23.0 (SPSS, Chicago, IL, USA) was used for data analysis. Details are described in the supplementary material.

\section{Results}

\section{Patients}

The baseline demographic and clinical characteristics of the 80 COPD patients from the BASCO study that were included in the discovery cohort are shown in supplementary table S1. This cohort includes patients with relevant smoking history, severe obstruction, mild hyperinflation, marked impairment of diffusing capacity of the lung for carbon monoxide (DLCO) and significant emphysema. All patients were adequately treated.

From the 638 patients that were recruited in the validation cohort, a total of 506 patients attended a scheduled visit at 6 months and were therefore eligible for inclusion in subsequent analyses (figure 1). The baseline demographic and clinical characteristics of the patients included in the validation cohort are shown in table 1 . Both cohorts were well matched. Most patients were male, had a considerable smoking history, clinically relevant disease (at least one exacerbation requiring physician attention in the previous year) and multiple comorbidities (supplementary table S2). At the stable-state visit, 155 patients (30.6\%) were able to produce sputum of good quality for microbiological analysis. From those, 48 samples (30.9\%) evidenced potentially pathogenic bacteria growth. Patients were followed during a median (interquartile range (IQR)) period of 722 (395-762) days. During the follow-up, 317 patients (62.6\%) suffered from at least one exacerbation and 38 patients $(7.5 \%)$ died. 
TABLE 1 Baseline demographic and clinical characteristics of patients included in the validation cohort (PROMISE-COPD study)

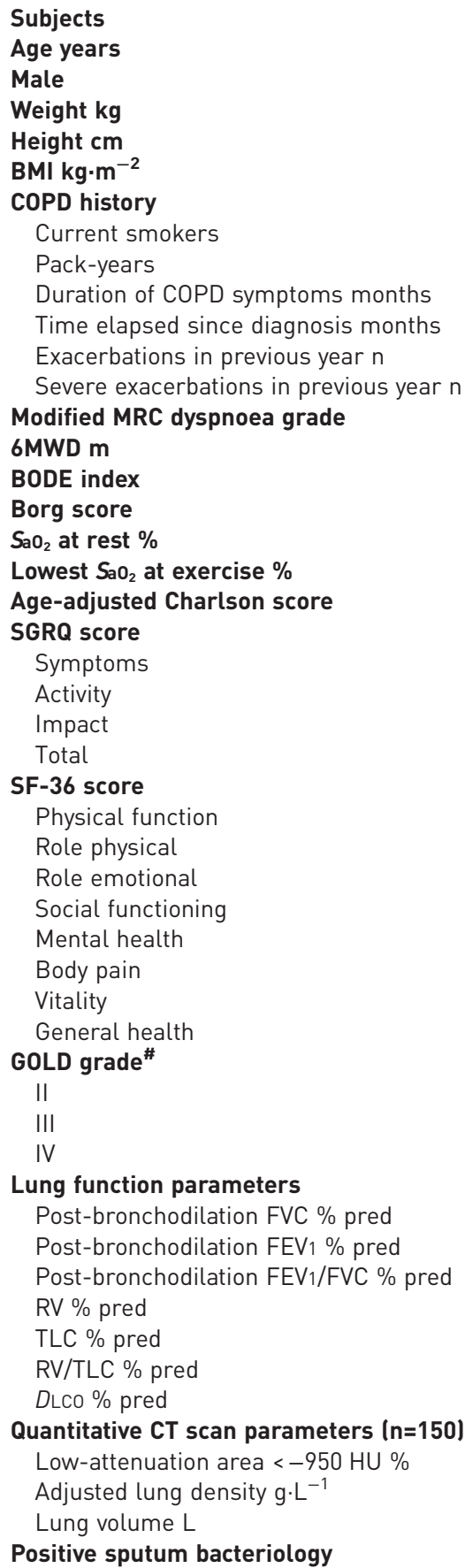

Data are presented as $\mathrm{n}$, mean $\pm \mathrm{SD}, \mathrm{n}(\%)$, median (interquartile range) or $\mathrm{n} / \mathrm{N}(\%)$. BMI: body mass index COPD: chronic obstructive pulmonary disease; MRC: Medical Research Council; 6MWD: 6-min walk distance; BODE: body mass, airflow obstruction, dyspnoea, exercise capacity; $\mathrm{SaO}_{2}$ : arterial oxygen saturation; SGRQ: St George's Respiratory Questionnaire; SF-36: Short Form-36; GOLD: Global Initiative for Chronic Obstructive Lung Disease; FEV1: forced expiratory volume in $1 \mathrm{~s}$; FVC: forced vital capacity; RV: residual volume; TLC: total lung capacity; DLCO: diffusing capacity of the lung for carbon monoxide; CT: computed tomography. " : GOLD grades are based on FEV1 \% pred: II, $\geqslant 50 \%$ to $<80 \%$; III, $\geqslant 30 \%$ to $<50 \%$; IV, $\leqslant 30 \%$. 
Patients that had an exacerbation performed an unscheduled visit and were sampled within 2 days (range $0-5$ days) after the event: $21.8 \%$ of patients were sampled on the same day, $16.1 \%$ of patients were sampled 1 day after the event, $14.0 \%$ of patients were sampled 2 days after the event, $10.1 \%$ of patients were sampled 3 days after the event, $8.3 \%$ of patients were sampled 4 days after the event and $5.7 \%$ of patients were sampled 5 days after the event.

The clinical outcomes within the 2-year follow-up period are shown in supplementary table S3. From baseline, median time to exacerbation was 338 days and to severe exacerbation was 701 days.

\section{HA is increased during COPD exacerbations}

Serum levels of HA were measured in the discovery cohort of 80 patients with COPD. Among these patients, 65 patients had stable disease and 15 patients had an exacerbation. The concentration of HA in serum was significantly higher in COPD patients at exacerbation compared with patients at stable state $(\mathrm{p}=0.015)$ (figure $2 \mathrm{a}$ ).

Subsequently, we evaluated HA serum concentrations in the validation cohort of 506 patients with COPD. HA was measured at stable state, at exacerbations (moderate and severe) and at follow-up (4 weeks after exacerbation). HA was significantly increased at moderate and severe exacerbations compared with the stable state $(\mathrm{p}<0.001$ for both comparisons) (figure $2 \mathrm{~b}$ ). At follow-up, serum levels of HA remained significantly higher compared with serum levels at stable state $(\mathrm{p}<0.001)$.

\section{Correlation analysis of HA serum levels with baseline parameters, markers of inflammation and} disease outcomes

HA levels in the serum of COPD patients at stable state were positively correlated with the age of patients $(\mathrm{p}<0.001)$, post-bronchodilation FEV1 \% pred $(\mathrm{p}=0.002)$, FEV $1 / \mathrm{FVC}(\mathrm{p}=0.025)$ and age-adjusted Charlson score $(p=0.001)$. Regarding markers of inflammation, HA serum levels were significantly correlated with adrenomedullin $(\mathrm{p}=0.001)$ and atrial natriuretic peptide $(\mathrm{p}<0.001)$ (table 2$)$. There was no significant correlation of serum levels of HA with body mass index, smoking history, Borg dyspnoea scale or walking distance. Furthermore, there was no significant correlation of HA with DLCO or with any of the quantitative CT scan parameters, indicating that HA is not related to emphysema.

Furthermore, HA was significantly associated $(\mathrm{p}<0.001)$ with time to death (HR 1.019 , 95\% CI 1.009 1.029). Cox regression multivariate analysis revealed that this association was independent of adjusted Charlson score, annual exacerbation rate and BODE index components (table 3).

Subsequently, in an exploratory analysis, we used linear mixed models to test the association between various clinical characteristics at baseline and the change of HA serum levels between the stable state and moderate or severe exacerbations. Among 102 baseline predictors tested, post-bronchodilation FEV1 \% pred was a significant predictor $(\mathrm{p}=0.0186)$ for HA increase at moderate exacerbations (supplementary table S4). Adjusted and unadjusted Charlson scores were significant predictors $(\mathrm{p}=0.0275$ and $\mathrm{p}=0.0328$, respectively) for HA increase at severe exacerbations (supplementary table S4).
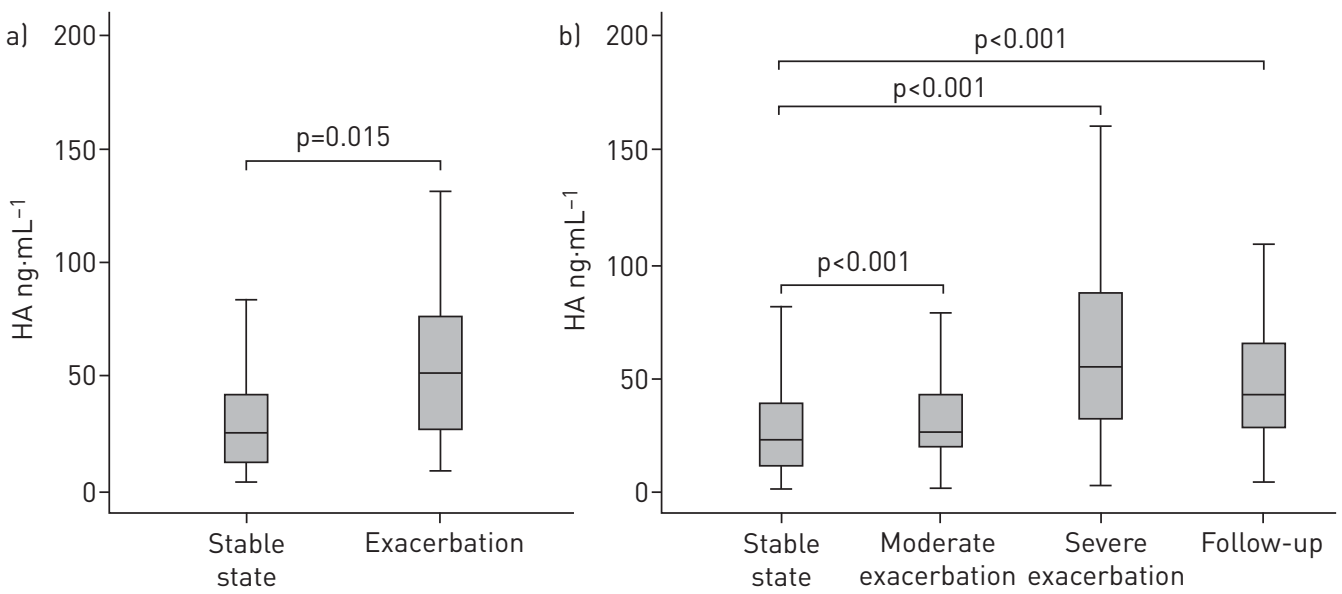

FIGURE 2 Hyaluronic acid (HA) in the serum of chronic obstructive pulmonary disease (COPD) patients. Serum concentration of HA was measured by ELISA in a) 80 participants of the discovery cohort (65 patients were at stable state and 15 patients had an exacerbation of COPD), and b) 506 participants of the validation cohort at stable state $(n=506)$, at moderate exacerbation $(n=219)$, at severe exacerbation $(n=88)$ and at 4 -week follow-up after an exacerbation ( $\mathrm{n}=92)$. All measurements were performed in duplicates. 
TABLE 2 Correlation analysis (Spearman's rho) of hyaluronic acid in serum of chronic obstructive pulmonary disease patients at stable state $(n=506)$ included in the validation cohort with baseline parameters

\begin{tabular}{|c|c|c|}
\hline & $\mathbf{r}$ & p-value \\
\hline Age & 0.233 & $<0.001$ \\
\hline Body mass index & 0.055 & 0.216 \\
\hline Pack-years & -0.078 & 0.086 \\
\hline Post-bronchodilation FEV 1 \% pred & 0.143 & 0.002 \\
\hline Post-bronchodilation FEV1/FVC & 0.103 & 0.025 \\
\hline Adjusted Charlson score & 0.149 & 0.001 \\
\hline Borg dyspnoea scale & 0.012 & 0.800 \\
\hline 6MWD & -0.027 & 0.556 \\
\hline Survival time & -0.139 & $<0.001$ \\
\hline D.co & 0.112 & 0.135 \\
\hline \multicolumn{3}{|l|}{ CT scan quantitative parameters } \\
\hline Low-attenuation area <-950 HU \% & -0.037 & 0.687 \\
\hline Adjusted lung density $\mathrm{g} \cdot \mathrm{L}^{-1}$ & 0.012 & 0.899 \\
\hline Lung volume $\mathrm{L}$ & -0.016 & 0.865 \\
\hline \multicolumn{3}{|l|}{ Inflammation markers } \\
\hline Procalcitonin $\mu \mathrm{g} \cdot \mathrm{L}^{-1}$ & 0.023 & 0.610 \\
\hline Adrenomedullin $\mathrm{nmol} \cdot \mathrm{L}^{-1}$ & 0.143 & 0.001 \\
\hline Copeptin pmol. $\mathrm{L}^{-1}$ & 0.053 & 0.239 \\
\hline Atrial natriuretic peptide $\mathrm{pmol} \cdot \mathrm{L}^{-1}$ & 0.173 & $<0.001$ \\
\hline
\end{tabular}

FEV1: forced expiratory volume in 1 s; FVC: forced vital capacity; 6MWD: 6-min walk distance; DLco: diffusing capacity of the lung for carbon monoxide; CT: computed tomography.

At exacerbation, HA serum levels were not significantly correlated with markers of systemic inflammation such as leukocyte counts and C-reactive protein (supplementary table S5).

\section{HA serum levels and sputum bacteriology}

At stable state, 155 patients could produce sputum of good quality for microbiological analysis that revealed potential pathogenic bacteria growth in 48 patients. Median (IQR) serum HA levels were similar in patients with positive cultures of potential pathogenic bacteria in sputum $\left(21.3(13.8-39.9) \mathrm{ng} \cdot \mathrm{mL}^{-1}\right)$ compared with patients without growth of potential pathogenic bacteria in sputum (19.0 $\left.(6.5-35.3) \mathrm{ng} \cdot \mathrm{mL}^{-1}\right)(\mathrm{p}=0.076)$. Similarly, there were no significant differences in median (IQR) HA serum levels at exacerbation between patients with positive sputum cultures and patients with negative sputum cultures at moderate exacerbations (26.1 (20.8-46.3) versus $\left.34.3(21.6-59.1) \mathrm{ng} \cdot \mathrm{mL}^{-1} ; \mathrm{p}=0.575\right)$ and at severe exacerbations (44.5 (28.8-83.6) versus $\left.83.2(44.3-104.2) \mathrm{ng} \cdot \mathrm{mL}^{-1} ; \mathrm{p}=0.362\right)$.

HYAL-1 in the serum of patients with COPD

As the biological effects of $\mathrm{HA}$ are determined by its molecular weight, we further investigated the enzymatic activity as well as protein levels of HYAL-1, which is the main enzyme that degrades HA, in serum. Enzymatic activity of HYAL in the serum of COPD patients at stable state was evident as a single lysis band that migrated with a molecular mass of $57 \mathrm{kDa}$ corresponding to HYAL-1 (figure $3 \mathrm{a}$ ). Quantification of the enzymatic lysis bands from serum of COPD patients from the validation cohort $(n=506)$ revealed that HYAL-1 enzymatic activity was inversely correlated with post-bronchodilation FEV1

TABLE 3 Multivariate cox regression analysis for time to death among the study population included in the validation cohort

\begin{tabular}{lcc} 
& HR (95\% CI) & p-value \\
\hline Hyaluronic acid & $1.019(1.009-1.029)$ & $<0.001$ \\
Adjusted Charlson score & $1.207(0.961-1.547)$ & 0.105 \\
Annual exacerbation rate & $1.306(1.037-1.644)$ & 0.023 \\
BODE index & $1.268(1.086-1.482)$ & 0.003 \\
\hline
\end{tabular}

BODE: body mass, airflow obstruction, dyspnoea, exercise capacity. 
$\%$ pred $(\mathrm{r}=-0.109, \mathrm{p}=0.034)$ and survival time $(\mathrm{r}=-0.118, \mathrm{p}=0.017)$ (figure $3 \mathrm{~b}$ and $\mathrm{c})$. There was no significant correlation of HYAL-1 activity with age, body mass index, smoking history, FEV1/FVC, Borg dyspnoea scale or walking distance (supplementary table S6).

HYAL-1 levels in serum of COPD patients at stable state were significantly correlated with age, and adjusted and unadjusted Charlson score $(\mathrm{p}<0.001$ for all), and inversely correlated with walking distance $(\mathrm{p}=0.001)$. Regarding markers of inflammation, HYAL-1 serum levels were significantly correlated with procalcitonin $(\mathrm{p}=0.011)$, adrenomedullin $(\mathrm{p}<0.001)$, copeptin $(\mathrm{p}=0.004)$ and atrial natriuretic peptide $(\mathrm{p}<0.001)$ (table 4). There was no significant correlation of HYAL-1 with DLCO or any of the quantitative CT scan parameters, indicating that enzymatic degradation of HA is not related to emphysema.

HYAL-1 serum levels were significantly increased at moderate exacerbations $(p=0.004)$ and severe exacerbations $(p=0.003)$ (figure 4). At follow-up, 4 weeks after exacerbations, HYAL-1 levels were significantly decreased to control levels ( $\mathrm{p}<0.001$ for moderate and for severe exacerbations).

\section{Effect of treatment on serum levels of HA and HYAL-1}

We further explored whether various treatments may alter serum levels of HA and HYAL-1 in COPD patients at stable state. As shown in table 5, median (IQR) HA serum levels did not differ between patients under treatment with inhaled corticosteroids (ICSs) $\left(21.7(10.1-38.8) \mathrm{ng} \cdot \mathrm{mL}^{-1}\right)$ and patients that were not receiving ICSs $\left(22.6(13.1-44.5) \mathrm{ng} \cdot \mathrm{mL}^{-1}\right)(\mathrm{p}=0.437)$. Furthermore, there were no significant differences in median (IQR) HA serum levels between patients that were receiving and patients that were not receiving treatment with systemic corticosteroids (20.0 (9.0-46.9) versus $\left.22.3(10.3-38.8) \mathrm{ng} \cdot \mathrm{mL}^{-1} ; \mathrm{p}=0.846\right)$ or with the combination of long-acting $\beta_{2}$-agonists (LABAs) and corticosteroids (21.2 (9.4-38.1) versus 23.3 (13.644.2) ng. $\left.\mathrm{mL}^{-1} ; \mathrm{p}=0.093\right)$. Similarly, long-acting muscarinic antagonists did not affect median (IQR) HA serum levels (21.2 (9.7-38.0) versus $\left.25.2(12.1-41.2) \mathrm{ng} \cdot \mathrm{mL}^{-1} ; \mathrm{p}=0.324\right)$.

Even when we adjusted for FEV1 \% pred, we were not able to detect any significant difference in HA serum levels between patients that were receiving or not receiving any of the aforementioned treatments. In addition, the use of systemic corticosteroids at moderate exacerbations was not significantly associated with HA levels after 4 weeks of follow-up as revealed by analysis in paired samples $(p=0.458)$.

Similarly, median (IQR) HYAL-1 serum levels did not differ between patients under treatment with ICSs $\left(1.7(1.2-2.4) \mathrm{ng} \cdot \mathrm{mL}^{-1}\right)$ and patients that were not receiving ICS $\left(1.6(1.1-2.4) \mathrm{ng} \cdot \mathrm{mL}^{-1}\right)(\mathrm{p}=0.632)($ table 5). Furthermore, there were no significant differences in median (IQR) HYAL-1 serum levels between
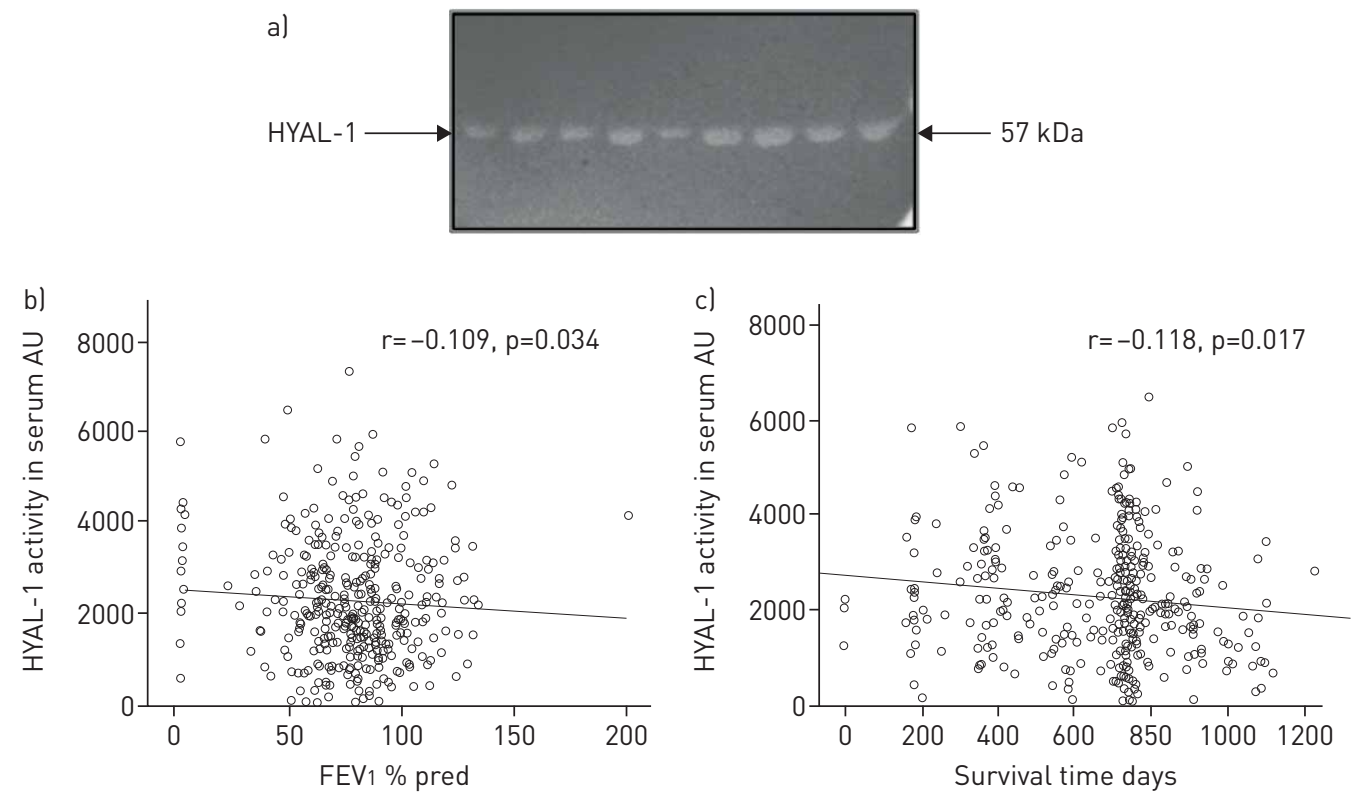

FIGURE 3 Hyaluronidase (HYAL)-1 activity in serum samples of chronic obstructive pulmonary disease (COPD) patients. FEV 1 : forced expiratory volume in $1 \mathrm{~s} ; \mathrm{HA}$ : hyaluronic acid. Enzymatic activity of HYAL-1 was assessed in serum samples ( $5 \mu \mathrm{L}$ from diluted serum 1/100) of COPD patients at stable state $(n=506)$ from the validation cohort by HA substrate zymography. a) Representative HA substrate zymography of serum samples obtained from nine patients with COPD at stable state. The lysis band migrated at $57 \mathrm{kDa}$ corresponds to HYAL-1 activity. b, c) Correlation (Spearman's rho) between values of HYAL-1 activity at stable state quantitated by the Kodak computer-assisted image analysis program and b) FEV $1 \%$ pred and c) survival time. 
TABLE 4 Correlation analysis (Spearman's rho) of hyaluronidase- 1 in serum of chronic obstructive pulmonary disease patients at stable state $(n=506)$ included in the validation cohort with baseline parameters

\begin{tabular}{|c|c|c|}
\hline & $\mathbf{r}$ & p-value \\
\hline Age & 0.298 & $<0.001$ \\
\hline Body mass index & 0.068 & 0.385 \\
\hline Pack-years & 0.028 & 0.724 \\
\hline Post-bronchodilation FEV $1 \%$ pred & 0.087 & 0.278 \\
\hline Adjusted Charlson score & 0.370 & $<0.001$ \\
\hline Charlson score & 0.311 & $<0.001$ \\
\hline Borg dyspnoea scale & 0.054 & 0.510 \\
\hline 6MWD & -0.275 & 0.001 \\
\hline Survival time & -0.137 & 0.080 \\
\hline DLco & 0.068 & 0.687 \\
\hline \multicolumn{3}{|l|}{ CT scan quantitative parameters } \\
\hline Low-attenuation area <-950 HU \% & -0.048 & 0.799 \\
\hline Adjusted lung density $\mathrm{g} \cdot \mathrm{L}^{-1}$ & 0.114 & 0.541 \\
\hline Lung volume $\mathrm{L}$ & 0.263 & 0.152 \\
\hline \multicolumn{3}{|l|}{ Inflammation markers } \\
\hline Procalcitonin $\mu \mathrm{g} \cdot \mathrm{L}^{-1}$ & 0.200 & 0.011 \\
\hline Adrenomedullin nmol. $\mathrm{L}^{-1}$ & 0.298 & $<0.001$ \\
\hline Copeptin pmol. $\mathrm{L}^{-1}$ & 0.227 & 0.004 \\
\hline Atrial natriuretic peptide $\mathrm{pmol} \cdot \mathrm{L}^{-1}$ & 0.298 & $<0.001$ \\
\hline
\end{tabular}

FEV1: forced expiratory volume in $1 \mathrm{~s}$; 6MWD: 6-min walk distance; DLCo: diffusing capacity of the lung for carbon monoxide; CT: computed tomography.

patients that were receiving and patients that were not receiving treatment with systemic corticosteroids (1.7 (1.4-2.6) versus $\left.1.7(1.2-2.4) \mathrm{ng} \cdot \mathrm{mL}^{-1} ; \mathrm{p}=0.637\right)$ or with the combination of LABAs and corticosteroids (1.7 (1.2-2.5) versus $\left.1.7(1.1-2.4) \mathrm{ng} \cdot \mathrm{mL}^{-1} ; \mathrm{p}=0.640\right)$. Similarly, long-acting muscarinic antagonists did not affect median (IQR) HYAL-1 serum levels (1.7 (1.2-2.4) versus $1.7(1.1-2.5) \mathrm{ng} \cdot \mathrm{mL}^{-1}$; $\mathrm{p}=0.936)$.

Even when we adjusted for FEV1 \% pred, we were not able to detect any significant difference in HYAL-1 serum levels between patients that were receiving or not receiving any of the aforementioned treatments.

\section{Association of HA and HYAL-1 with biomarkers of ECM turnover}

We further investigated the association of HA and HYAL-1 with molecules reflecting ECM turnover, including fragments of collagen types I, III, IV and VI, pro-forms of collagen types III, V and VI, and neutrophil elastase-generated fragments of elastin (table 6). Serum levels of HA were significantly correlated with serum levels of pro-forms of collagen type III and type VI ( $p<0.001$ for both).

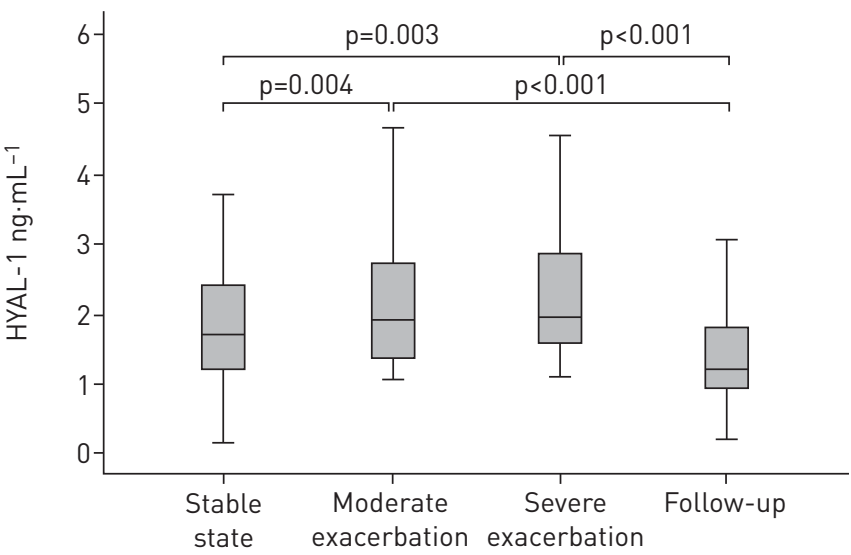

FIGURE 4 Hyaluronidase (HYAL)-1 in the serum of chronic obstructive pulmonary disease patients from the validation cohort. Serum concentration of HYAL-1 was measured by ELISA in 506 participants of the validation cohort at stable state $(n=506)$, at moderate exacerbation $(n=219)$, at severe exacerbation $(n=88)$ and at 4 -week follow-up after an exacerbation ( $\mathrm{n}=92)$. All measurements were performed in duplicates. 


\begin{tabular}{|c|c|c|c|c|}
\hline & $\mathrm{HA} \mathrm{ng} \cdot \mathrm{mL}^{-1}$ & p-value & HYAL-1 $\mathrm{ng} \cdot \mathrm{mL}^{-1}$ & p-value \\
\hline \multicolumn{5}{|c|}{ Inhaled corticosteroids } \\
\hline Yes & $21.7(10.1-38.8)$ & \multirow[t]{2}{*}{0.437} & $1.7(1.2-2.4)$ & \multirow[t]{2}{*}{0.632} \\
\hline No & $22.6(13.1-44.5)$ & & $1.6(1.1-2.43)$ & \\
\hline \multicolumn{5}{|c|}{ Systemic corticosteroids } \\
\hline Yes & $20.0(9.0-46.9)$ & \multirow[t]{2}{*}{0.846} & $1.7(1.4-2.6)$ & \multirow[t]{2}{*}{0.637} \\
\hline No & $22.3(10.3-38.8)$ & & $1.7(1.2-2.4)$ & \\
\hline \multicolumn{5}{|c|}{ Long-acting $\boldsymbol{\beta}_{2}$-agonists+corticosteroids } \\
\hline Yes & $21.2(9.4-38.1)$ & \multirow[t]{2}{*}{0.093} & $1.7(1.2-2.5)$ & \multirow[t]{2}{*}{0.640} \\
\hline No & $23.3(13.6-44.2)$ & & $1.7(1.1-2.4)$ & \\
\hline \multicolumn{5}{|c|}{ Long-acting muscarinic antagonists } \\
\hline Yes & $21.2(9.7-38.0)$ & \multirow[t]{2}{*}{0.324} & $1.7(1.2-2.4)$ & \multirow[t]{2}{*}{0.936} \\
\hline No & $25.2(12.1-41.2)$ & & $1.7(1.1-2.5)$ & \\
\hline
\end{tabular}

Data are presented as median (interquartile range), unless otherwise stated.

HYAL-1 levels were significantly associated with the pro-form of collagen type VI, and with the degradation fragments of collagen type VI $(p=0.012)$ and the $\alpha_{3}$ chain of collagen type IV $(p=0.020)$.

\section{Discussion}

In the present study, we investigated serum levels of HA in two independent, well-matched cohorts of COPD patients, and we report that HA is associated with COPD severity and outcome. To the best of our knowledge, this is the first study to show that HA and its degradation products resulting from the action of HYAL-1 may play a role in COPD, and that disease activity and subsequent outcome can be envisaged through detection of circulating HA. The current analysis provides new insights into the pathophysiology of COPD.

HA is a naturally occurring component of the ECM and it participates in a number of biological processes, such as cell migration and proliferation, water homeostasis, tissue repair, inflammation, cellmatrix signalling, and angiogenesis [25]. HA has exceptional hydrophilic characteristics and can bind $\sim 1000$ times its weight in water. Therefore, in the ECM, HA produces highly viscous gels that play an essential role in tissue homeostasis and biomechanical integrity [12]. HA can bind to specific cell surface receptors and induce intracellular signal transduction, but also creates a matrix coat around the cells and protects them from the environment.

In the present study, we provide evidence that serum levels of HA at stable state were significantly associated with time to death independently of adjusted Charlson score, annual exacerbation rate and BODE index components. It has been shown that HA serum levels are elevated in patients with dyslipidaemia and high cardiovascular risk [26]. In our study, patients included in the validation cohort had numerous comorbidities, including arterial hypertension and coronary arterial disease. However, the fact that the association of HA with time to death was independent of age-adjusted Charlson score

TABLE 6 Correlation analysis (Spearman's rho) of hyaluronic acid (HA) and hyaluronidase (HYAL)-1 in serum of chronic obstructive pulmonary disease patients at stable state $(n=506)$ included in the validation cohort with biomarkers of extracellular matrix turnover

\begin{tabular}{|c|c|c|c|c|}
\hline & \multicolumn{2}{|c|}{ HA } & \multicolumn{2}{|c|}{ HYAL-1 } \\
\hline & $\mathbf{r}$ & p-value & $\mathbf{r}$ & p-value \\
\hline Pro-collagen type III & 0.204 & $<0.001$ & 0.131 & 0.104 \\
\hline Pro-collagen type $\mathrm{V}$ & -0.057 & 0.251 & 0.060 & 0.490 \\
\hline Pro-collagen type VI & 0.182 & $<0.001$ & 0.270 & 0.001 \\
\hline Collagen type I degradation products & -0.047 & 0.349 & 0.002 & 0.979 \\
\hline Collagen type III degradation products & -0.001 & 0.979 & 0.120 & 0.136 \\
\hline Collagen type IV degradation products ( $\alpha_{1}$ chain) & -0.035 & 0.482 & 0.053 & 0.550 \\
\hline Collagen type IV degradation products ( $\alpha_{3}$ chain) & -0.063 & 0.206 & 0.203 & 0.020 \\
\hline Collagen type VI degradation products & -0.015 & 0.738 & 0.201 & 0.012 \\
\hline Neutrophil elastase-generated fragments of elastin & -0.004 & 0.932 & 0.114 & 0.158 \\
\hline
\end{tabular}


warrants that the observed association of HA with COPD outcome is not an epiphenomenon and that circulating HA in COPD patients can serve as an independent predictor for survival.

Accurate prediction of mortality is very important to identify COPD patients who would benefit by the implementation of intensified therapeutic measures, such as lung transplantation [27]. Several studies have attempted to find predictors of survival for COPD patients and explored the association of clinical characteristics with specific biomarkers of disease activity and/or progression [28, 29]. Several serum biomarkers have been described to be independently associated with increased risk of death [30, 31]. A few biomarkers have been shown to further contribute to predict mortality when added to known clinical scores such as the BODE index $[28,32,33]$. Since COPD is a complex and heterogeneous disease with various phenotypes, a panel of prognostic biomarkers reflecting the associated comorbidities and different pathobiological pathways that may be altered in this disease has been proposed [29, 31, 34]. Simultaneously elevated levels of adrenomedullin, arginine vasopressin and atrial natriuretic peptide have been shown to be associated with increased risk of death in patients with stable COPD [34]. In the present study, we provide evidence that HA is significantly correlated with adrenomedullin and atrial natriuretic peptide, and that HYAL-1 is correlated with adrenomedullin, copeptin, procalcitonin and atrial natriuretic peptide. To the best of our knowledge, this is the first study showing a correlation of HA and its degrading enzymes with these COPD prognostic biomarkers. It remains to be elucidated whether the concomitant estimation of HA and HYAL-1 together with well-established mortality predictors would contribute to increase the accuracy for the prediction of mortality.

We have previously shown that increased HA degradation in the bronchoalveolar lavage of COPD patients is associated with airway obstruction and not with emphysema [17]. The lack of a significant association between serum levels of HA and HYAL-1 with DLCO or with quantitative CT scan parameters representing the status of lung parenchyma that we observe in the present study is a further indication that HA and HYAL-1 are not related to emphysema. It remains to be elucidated whether HA degradation is associated with destruction of the terminal and transitional bronchioles, which have been recently shown to represent the initial site of injury in COPD even at an early stage of the disease $[35,36]$. However, such an analysis would require a novel multiresolution CT imaging protocol, not available in the present study.

In the lung, $\mathrm{HA}$ is a dynamic molecule with physiological functions that are closely related to its molecular weight. There is evidence that low-molecular-weight HA produced during tissue injury increases inflammatory responses, whereas the physiologically occurring high-molecular-weight HA is protective and has anti-inflammatory effects [10-12]. Here, we provide evidence, for the first time, that HYAL activity in the serum of 506 COPD patients was negatively correlated with FEV1 \% pred and with survival time. These results indicate that enzymatic degradation of physiologically occurring high-molecular-weight $\mathrm{HA}$ in COPD patients is associated with airflow limitation, impairment of lung function and time to death. In agreement with these data, previous studies have shown that short fragments of HA were detected in the bronchoalveolar lavage fluid [7, 17, 37-39] and in the parenchyma [7] in a number of lung diseases, indicating that HA turnover is a major component for the development, progression and resolution of inflammatory lung diseases [7, 17].

In addition to HA, degradation products of other ECM molecules are also associated with clinically relevant outcomes in COPD. Pro-forms and fragments of collagens have been shown to play a significant role in COPD severity and disease outcome $[18,24]$. In the present study, we show that serum levels of HA were significantly associated with pro-collagen types III and VI, the major collagens found in the lung interstitium and in large bronchi. HYAL-1 serum levels were associated with pro-collagen type VI as well as with the degradation products of collagen type IV and collagen type VI that is mainly found in the basement membranes. These results indicate that HA, in line with collagens, is a part of the increased ECM turnover in COPD, a process that determines disease severity and clinically relevant outcomes.

Exacerbations of COPD are important events in the natural history of the disease as they impact on disease progression, health status and survival. Therefore, there is an unmet need to understand the pathophysiological mechanisms involved in exacerbations of COPD and to identify molecules that are associated with airflow limitation during exacerbations. In the present study, we provide evidence that serum levels of HA are significantly increased at moderate and severe exacerbations of COPD compared with the stable state of the disease. HA levels at exacerbation were not related to bacterial infections or to markers of acute inflammation such as leukocyte counts and C-reactive protein. Concomitantly, HYAL-1 serum levels are also increased at exacerbation, indicating that COPD exacerbations are associated with increased degradation of HA leading to the formation of short HA fragments. In the last two decades extensive research has shown that signalling by short HA fragments contributes to the accumulation of immune cells in inflammatory sites [40-42], and the subsequent release of pro-inflammatory cytokines and metalloelastases $[43,44]$. 
Another interesting finding in our study was the fact that 4 weeks after exacerbations, HA serum levels remained significantly higher compared with the stable state, even though HYAL-1 levels were decreased to stable-state levels. Therefore, it is tempting to hypothesise that the termination of the action of HYAL-1 precedes the removal of the short fragments of HA from the circulation.

Our study has a few limitations. Measurements of HA serum concentration by ELISA do not provide information about the size of HA. Unfortunately, to date, there is no appropriate methodology to estimate the size of such a large glycosaminoglycan in serum samples. Thus, parallel measurements of HYAL-1, which is the main HYAL detected in human serum, provide the best indication for the size of HA. Another limitation of our study is that HA serum levels could be modified by treatment, since it has been shown that HA turnover is altered by glucocorticoids and LABAs [45-47], and the majority of COPD patients that participated in the present study were under treatment with ICSs ( $81 \%$ of the patients) or with ICS+LABA combination (61.3\% of the patients). However, when we stratified the analysis of HA and HYAL-1 according to treatment, even if the groups that were receiving treatment and the groups that were not receiving treatment were not balanced for numbers of patients, we could not observe any significant differences in HA or HYAL-1 levels between the groups receiving ICSs, systemic corticosteroids or ICS $+\mathrm{LABA}$ and the groups that were not receiving any treatment.

A major strength of our study is the large sample size and the comprehensive clinical characterisation of the patients that enabled us to establish that the association of HA with time to death was independent of adjusted Charlson score, annual exacerbation rate and BODE index components. A further strength relates to the two-stage design with two well-matched cohorts. The discovery cohort was designed to maximise the chances to establish HA as a potential molecule associated with disease severity and outcome, and to minimise unnecessary waste of patient samples. Additional strengths are the longitudinal sampling undertaken at three points in time (at stable state, at exacerbation and at follow-up) and the longitudinal clinical data that enabled us to determine disease outcomes.

In conclusion, the results of our study indicate that HA is associated with COPD severity and outcome, and predicts overall survival. During COPD exacerbations, enzymatic degradation of HA is associated with airflow limitation and impairment of lung function. Thus, HA and its degrading enzyme HYAL-1 may be proved to serve as potential targets to control airway inflammation and remodelling in COPD.

Conflict of interest: None declared.

Support statement: This study was funded by Schweizerischer Nationalfonds zur Förderung der Wissenschaftlichen Forschung (PP00-P3_128412/1), Swiss Lungenliga (2015-18) and Universität Basel (DMS 2182). Funding information for this article has been deposited with the Crossref Funder Registry.

\section{References}

1 Seemungal TA, Donaldson GC, Paul EA, et al. Effect of exacerbation on quality of life in patients with chronic obstructive pulmonary disease. Am J Respir Crit Care Med 1998; 157: 1418-1422.

2 Stolz D, Hirsch HH, Schilter D, et al. Intensified therapy with inhaled corticosteroids and LABA at the onset of URTI to prevent COPD exacerbations - a multicentre, randomised, double-blind, placebo-controlled trial. Am J Respir Crit Care Med 2018; 197: 1136-1146.

3 Postma DS, Timens W. Remodeling in asthma and chronic obstructive pulmonary disease. Proc Am Thorac Soc 2006; 3: 434-439.

4 Allegra L, Della Patrona S, Petrigni G. Hyaluronic acid: perspectives in lung diseases. Handb Exp Pharmacol 2012; 207: 385-401.

5 Kakehi K, Kinoshita M, Yasueda S. Hyaluronic acid: separation and biological implications. J Chromatogr B Analyt Technol Biomed Life Sci 2003; 797: 347-355.

6 Itano N, Sawai T, Yoshida M, et al. Three isoforms of mammalian hyaluronan synthases have distinct enzymatic properties. J Biol Chem 1999; 274: 25085-25092.

7 Papakonstantinou E, Kouri FM, Karakiulakis G, et al. Increased hyaluronic acid content in idiopathic pulmonary arterial hypertension. Eur Respir J 2008; 32: 1504-1512.

8 Papakonstantinou E, Karakiulakis G. The 'sweet' and 'bitter' involvement of glycosaminoglycans in lung diseases: pharmacotherapeutic relevance. Br J Pharmacol 2009; 157: 1111-1127.

9 Stern R, Asari AA, Sugahara KN. Hyaluronan fragments: an information-rich system. Eur J Cell Biol 2006; 85: 699-715.

10 Deed R, Rooney P, Kumar P, et al. Early-response gene signalling is induced by angiogenic oligosaccharides of hyaluronan in endothelial cells. Inhibition by nonangiogenic, high-molecular-weight hyaluronan. Int J Cancer 1997; 71: 251-256

11 Rooney P, Wang M, Kumar P, et al. Angiogenic oligosaccharides of hyaluronan enhance the production of collagens by endothelial cells. J Cell Sci 1993; 105: 213-218.

12 Toole BP. Hyaluronan: from extracellular glue to pericellular cue. Nat Rev Cancer 2004; 4: 528-539.

13 Cantor JO, Cerreta JM, Ochoa M, et al. Therapeutic effects of hyaluronan on smoke-induced elastic fiber injury: does delayed treatment affect efficacy? Lung 2011; 189: 51-56. 
14 Jiang D, Liang J, Fan J, et al. Regulation of lung injury and repair by Toll-like receptors and hyaluronan. Nat Med 2005; 11: 1173-1179.

15 Petrigni G, Allegra L. Aerosolised hyaluronic acid prevents exercise-induced bronchoconstriction, suggesting novel hypotheses on the correction of matrix defects in asthma. Pulm Pharmacol Ther 2006; 19: 166-171.

16 Klagas I, Goulet S, Karakiulakis G, et al. Decreased hyaluronan in airway smooth muscle cells from patients with asthma and COPD. Eur Respir J 2009; 34: 616-628.

17 Papakonstantinou E, Roth M, Klagas I, et al. COPD exacerbations are associated with pro-inflammatory degradation of hyaluronic acid. Chest 2015; 148: 1497-1507.

18 Stolz D, Leeming DJ, Kristensen JH, et al. Systemic biomarkers of collagen and elastin turnover are associated with clinically relevant outcomes in COPD. Chest 2017; 151: 47-59.

19 Mandal J, Roth M, Papakonstantinou E, et al. Treatment with long acting muscarinic antagonists stimulates serum levels of irisin in patients with COPD. Pulm Pharmacol Ther 2018; 48: 111-116.

20 Boeck L, Soriano JB, Brusse-Keizer M, et al. Prognostic assessment in COPD without lung function: the B-AE-D indices. Eur Respir J 2016; 47: 1635-1644.

21 Mandal J, Malla B, Steffensen R, et al. Mannose-binding lectin protein and its association to clinical outcomes in COPD: a longitudinal study. Respir Res 2015; 16: 150.

22 Abramoff MD, Magalhaes PJ, Ram SJ. Abramoff: image processing with ImageJ. Biophotonics 2004; 11: 36-42.

23 Chapman KR, Burdon JGW, Piitulainen E, et al. Intravenous augmentation treatment and lung density in severe $\alpha 1$ antitrypsin deficiency (RAPID): a randomised, double-blind, placebo-controlled trial. Lancet 2015; 386: 360-368.

24 Schumann MD, Leeming D, Papakonstantinou E, et al. Collagen degradation and formation are elevated in exacerbated COPD compared with stable disease. Chest 2018; 154: 798-807.

25 Garantziotis S, Brezina M, Castelnuovo P, et al. The role of hyaluronan in the pathobiology and treatment of respiratory disease. Am J Physiol Lung Cell Mol Physiol 2016; 310: L785-L795.

26 Papanastasopoulou C, Papastamataki M, Karampatsis P, et al. Cardiovascular risk and serum hyaluronic acid: a preliminary study in a healthy population of low/intermediate risk. J Clin Lab Anal 2017; 31: e22010.

27 Reed RM, Cabral HJ, Dransfield MT, et al. Survival of lung transplant candidates with COPD: BODE score reconsidered. Chest 2018; 153: 697-701.

28 Celli BR, Locantore N, Yates J, et al. Inflammatory biomarkers improve clinical prediction of mortality in chronic obstructive pulmonary disease. Am J Respir Crit Care Med 2012; 185: 1065-1072.

29 Kelly E, Owen CA, Pinto-Plata V, et al. The role of systemic inflammatory biomarkers to predict mortality in chronic obstructive pulmonary disease. Expert Rev Respir Med 2013; 7: 57-64.

30 Bernasconi M, Tamm M, Bingisser R, et al. Midregional proatrial natriuretic peptide predicts survival in exacerbations of COPD. Chest 2011; 140: 91-99.

31 Stolz D, Christ-Crain M, Morgenthaler NG, et al. C-reactive protein, and procalcitonin as prognostic biomarkers in acute exacerbation of COPD. Chest 2007; 131: 1058-1067.

32 Stolz D, Kostikas K, Blasi F, et al. Adrenomedullin refines mortality prediction by the BODE index in COPD: the "BODE-A" index. Eur Respir J 2014; 43: 397-408.

33 Stolz D, Christ-Crain M, Morgenthaler NG, et al. Plasma pro-adrenomedullin but not plasma pro-endothelin predicts survival in exacerbations of COPD. Chest 2008; 134: 263-272.

34 Stolz D, Meyer A, Rakic J, et al. Mortality risk prediction in COPD by a prognostic biomarker panel. Eur Respir J 2014; 44: 1557-1570

35 Koo HK, Vasilescu DM, Booth S, et al. Small airways disease in mild and moderate chronic obstructive pulmonary disease: a cross-sectional study. Lancet Respir Med 2018; 6: 591-602.

36 Russell DW, Wells JM. COPD ground zero: small airways rather than alveoli as the initial site of injury. Lancet Respir Med 2018; 6: 568-569.

37 Bracke KR, Dentener MA, Papakonstantinou E, et al. Enhanced deposition of low-molecular-weight hyaluronan in lungs of cigarette smoke-exposed mice. Am J Respir Cell Mol Biol 2010; 42: 753-761.

38 Garantziotis S, Li Z, Potts EN, et al. Hyaluronan mediates ozone-induced airway hyperresponsiveness in mice. $J$ Biol Chem 2009; 284: 11309-11317.

39 Liang J, Jiang D, Jung Y, et al. Role of hyaluronan and hyaluronan binding proteins in human asthma. J Allergy Clin Immunol 2011; 128: 403-411.

40 McDonald B, McAvoy EF, Lam F, et al. Interaction of CD44 and hyaluronan is the dominant mechanism for neutrophil sequestration in inflamed liver sinusoids. J Exp Med 2008; 205: 915-927.

41 Savani RC, Hou G, Liu P, et al. A role for hyaluronan in macrophage accumulation and collagen deposition after bleomycin induced lung injury. Am J Respir Cell Mol Biol 2000; 23: 475-484.

42 Zaman A, Cui Z, Foley JP, et al. Expression and role of the hyaluronan receptor RHAMM in inflammation after bleomycin injury. Am J Respir Cell Mol Biol 2005; 33: 447-454.

43 McKee CM, Penno MB, Cowman M, et al. Hyaluronan (HA) fragments induce chemokine gene expression in alveolar macrophages. The role of HA size and CD44. J Clin Invest 1996; 98: 2403-2413.

44 Horton MR, Shapiro S, Bao C, et al. Induction and regulation of macrophage metalloelastase by hyaluronan fragments in mouse macrophages. J Immunol 1999; 162: 4171-4176.

45 Deshpande M, Papp S, Schaffer L, et al. Hydrocortisone and triiodothyronine regulate hyaluronate synthesis in a tissue-engineered human dermal equivalent through independent pathways. J Biosci Bioeng 2015; 119: 226-236.

46 Papakonstantinou E, Klagas I, Karakiulakis G, et al. Glucocorticoids and $\beta_{2}$-agonists regulate the pathologic metabolism of hyaluronic acid in COPD. Pulm Pharmacol Ther 2018; 48: 104-110.

47 Papakonstantinou E, Klagas I, Karakiulakis G, et al. Steroids and $\beta_{2}$-agonists regulate hyaluronan metabolism in asthmatic airway smooth muscle cells. Am J Respir Cell Mol Biol 2012; 47: 759-767. 